\title{
Analysis of Mental State of Patients after Drug Addiction and Withdrawal Guided by PETCT Image Based on Optimized Image Fusion Algorithm
}

\author{
Yaowen Pang ${ }^{1}{ }^{1}$ and Xiang Peng $\left(\mathbb{D}^{2}\right.$ \\ ${ }^{1}$ Department of Radiology, Brain Hospital of Hunan Province, Changsha 410000, Hunan, China \\ ${ }^{2}$ Department of PET/CT Center, Hunan Cancer Hospital, Changsha 410013, Hunan, China \\ Correspondence should be addressed to Xiang Peng; pengxiang@hnca.org.cn
}

Received 19 June 2021; Accepted 8 September 2021; Published 4 October 2021

Academic Editor: Gustavo Ramirez

Copyright (c) 2021 Yaowen Pang and Xiang Peng. This is an open access article distributed under the Creative Commons Attribution License, which permits unrestricted use, distribution, and reproduction in any medium, provided the original work is properly cited.

\begin{abstract}
Blood oxygen level-dependent functional magnetic resonance imaging (BOLD-fMRI) studies have shown that drug-dependent patients are activated in different addictive brain areas under the stimulation of relevant environmental cues, which in turn leads to craving and relapse. This study uses magnetic resonance spectroscopy to measure brain temperature to explore the brain temperature changes in different addictive brain regions of heroin and methamphetamine addicts in a short-term withdrawal state and to explore whether the quantitative index of brain temperature change can be used as a diagnostic drug Methods. The subjects were scanned by resting-state MRI spectroscopy first and then subjected to MRI spectroscopy scanning under visual stimulation. The subjects were required to watch the heroin/meth-related clue pictures carefully during visual stimulation. The measured chemical shift value of N-acetyl-aspartic acid (NAA) is substituted into the brain temperature calculation formula $T=37+100$ to obtain the brain temperature before and after visual stimulation. In addition, the anxiety and depression states of heroin and methamphetamine-dependent patients were evaluated. Results. There was no statistically significant change in the brain temperature of the prefrontal cortex before and after visual stimulation in heroin and methamphetamine-dependent subjects; compared with the normal group, there was no change in prefrontal cortex brain temperature before and after visual stimulation in heroin and methamphetamine-dependent subjects. Statistical Significance. The changes of hippocampal temperature before and after visual stimulation in methamphetamine-dependent patients were not statistically significant; compared with the normal group, there was no statistically significant difference in the changes of hippocampal temperature before and after visual stimulation in methamphetamine-dependent patients. Conclusion. This study initially found that the visual cues related to heroin and methamphetamine were not enough to cause significant changes in the brain temperature of the prefrontal cortex.
\end{abstract}

\section{Introduction}

Drug dependence can produce corresponding physiological, cognitive, and behavioural symptoms. Dependents are aware of the harm caused by addictive substances but still involuntarily continue to use them, leading to increased drug tolerance and inducing impulsive drug-seeking behaviour, resulting in serious withdrawal symptoms. Drug dependence has the characteristics of chronic and recurrent episodes. In addition, drug-dependent persons in the withdrawal period are prone to craving and relapse under the induction of drug clues. Therefore, how to completely eliminate drug addiction has become a difficult point in clinical treatment.

Drug abuse and dependence are also a worldwide public health problem, which seriously threatens human life and health. The abuse of heroin, the traditional drug, has become the most serious public health problem in China. Its addiction can extensively damage the neurocognitive functions of the brain, including damage to inhibitory 
control, cognitive flexibility, working memory, and decision-making functions. In foreign countries, new drugs (synthetic drugs) represented by methamphetamine (meth) are mainly used. In recent years, the use of methamphetamine has become more widespread and has become a focus of global concern. It has become a new serious threat to China's drug control. The social and economic problems caused by this have become more and more serious, which also poses greater challenges to drug treatment. However, the current drug addiction mechanism and pathological mechanism have not been clearly studied, and drug treatment measures are not perfect. The establishment of successful drug treatment programs is often limited due to insufficient understanding of drug damage to the brain tissue structure and function, and there is no objective technology to evaluate the psychological craving and the effect of drug addicts. At present, it is possible to study the neurological mechanism of drugs affecting brain tissue and function at the molecular and genetic level, but it is limited to focal and isolated tissue specimens. The information revealed by the results is still difficult to explain the complex effects of drugs on the brain [1].

Magnetic resonance spectroscopy imaging can noninvasively detect brain metabolite concentration and brain temperature changes in vivo and can be repeated many times. It has great advantages in the study of brain structure and brain function. Based on reports in the literature on animal experiments, injecting drugs can significantly increase brain temperature. Therefore, this study uses the effects of drugs on the structure and function of brain tissues and changes in brain temperature as the entry point and intends to use magnetic resonance spectroscopy (MRS) to monitor drug production. This paper provides theoretical basis for clinical drug rehabilitation of drug addicts by studying the changes of brain temperature under the relevant visual stimuli, objectively evaluating the degree of craving of drug addicts through the changes of brain temperature, and scientifically evaluating the psychological desire of their family members.

\section{Materials and Methods}

2.1. Research Object. The subjects of this experiment are 14 heroin addicts (HD) and 12 methamphetamine-dependent (MD) and 28 healthy volunteers (HC) (because of the different visual stimulation pictures of heroin and methamphetamine in the experiment, healthy volunteers were randomized and divided into two groups, of which 16 matched the heroin group and 12 matched the methamphetamine group). The drug dependents were all patients receiving compulsory drug treatment in the Special Drug Rehabilitation Hospital of the Public Security Bureau. Healthy volunteers used posters in hospitals and communities. Inclusion criteria included the following: (1) both sexes are available; (2) age is $18-45$; (3) they are righthanded; (4) they meet the diagnostic criteria for heroin and methamphetamine dependence in DSM-IV, and the urine test was positive upon admission; (5) both the heroin group and the methamphetamine group are currently in a withdrawal state, and the withdrawal time is less than 6 months; (6) except for heroin or methamphetamine dependence, there are no obvious other mental diseases, no serious heart, liver, kidney, and nervous system diseases; no psychotropic drugs are used; (7) they meet the MRI safety scan standard; (8) the naked eye vision is above 1.0. Exclusion criteria included the following: (1) patients with severe heart, liver, kidney, HIV, and other diseases; (2) patients with cognitive dysfunction, depression, and other neuropsychiatric diseases; (3) patients with a history of central nervous system; (4) colour blindness and colour weak patients; (5) high fever and MR scan contraindications.

2.2. Experimental Steps. The experiment is divided into two stages. Preparation stage: participants need to understand the content and process of the experiment, sign an informed consent form at the same time, and then fill out a questionnaire about general survey of anxiety, depression, and drug use. Participants received MRI simulation scan training before the experiment to adapt to the experimental environment as soon as possible. Experimental phase: all subjects' scans are completed between 13:00 and 18:00. Before the start of the experiment, the subject was escorted to the MR scanning room, and the subject was asked to lie flat in the scanner. In order to minimize the noise interference of magnetic resonance equipment, all subjects are required to wear earplugs and earphones, and at the same time, fix the subject's head with a foam pad. During the scan, the subjects were asked to keep their heads still, relax their bodies, keep quiet, and concentrate. First, perform a single voxel 1HMRS scan for each subject at rest (the subject stays awake, eyes closed, and quiet). The scanning site is the prefrontal cortex and bilateral hippocampus; then, the subject is scanned for 1HMRS under visual stimulation (the subject remained awake, eyes open, and quiet), and the subject was asked to watch the pictures carefully. During the experiment, the ambient temperature of the MR scanning room was monitored and kept at about $20^{\circ} \mathrm{C}$. Before and after the experiment, the same professional psychologist provided psychological counselling to all subjects [2].

2.3. Experimental Design. The MRS area of interest is the prefrontal lobe and bilateral hippocampus related to the reward loop (see Figures 1-3). The prefrontal cortex area of interest (VOI) selects the clearest level of the caudate nucleus on the axial T2WI image as its location map. The voxel location frame is placed across the midline including the left and right hemisphere prefrontal cortex, and the posterior boundary reaches the corpus callosum. The VOI is $1 \mathrm{~cm}^{3}$ in front of the mouth. The hippocampal VOI is located at the largest level of the hippocampus in the T2-FLAIR sequence on the oblique coronal plane. The scope can include the hippocampus and its lateral temporal lobe white matter. The size of the VOI is $4.5 \mathrm{~cm}^{3}$.

Using point-resolved spectroscopy (PRESS), scanning parameters are $\mathrm{TE}=144 \mathrm{~ms}, \mathrm{TR}=2000 \mathrm{~ms}$, prefrontal $\mathrm{NSA}=128$, and hippocampus NSA $=208$. Three-plane 

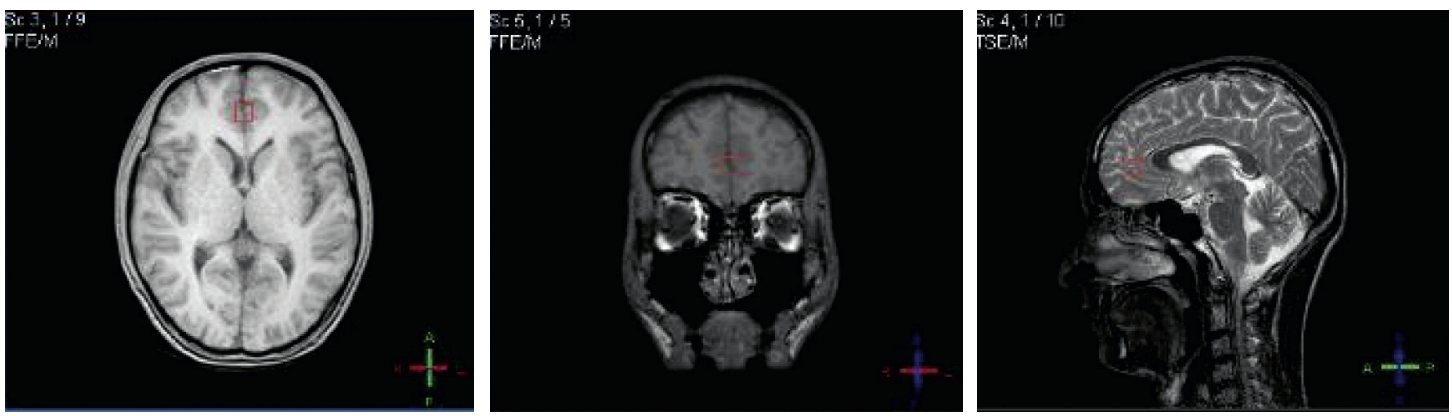

Figure 1: Location map of the prefrontal cortex.

positioning is selected, and the intravoxel shimming is automatically completed. When the maximum full width (FWHM) is less than $13 \mathrm{~Hz}$, the image is collected and the water suppression image is collected. When the suppression level reaches 99\%, the spectrum data is collected. The spectral curve and the chemical shift of NAA are automatically generated after the end of the scan (see Figures 4-8).

2.4. Equipment and Scanning Parameters. This experiment uses PHILIPSMRSystemsAchieva1.5TNOVADUAL superconducting magnetic resonance scanner and an 8-channel head quadrature coil. The scanning sequence and parameters are as follows: (1) T1WItra: minimum TR, minimum TE, FOVAP $230 \mathrm{~mm}$, RL $183 \mathrm{~mm}$, FH $125 \mathrm{~mm}$, layer thickness $6 \mathrm{~mm}$, layer number 18, and layer spacing $1 \mathrm{~mm}$; (2) T2WIsag: TR minimum value, TE $100 \mathrm{~ms}$, FOVAP $231 \mathrm{~mm}$, RL $109 \mathrm{~mm}, \mathrm{FH} 270 \mathrm{~mm}$, layer thickness $5 \mathrm{~mm}$, layer number 18 , and layer spacing $1.1 \mathrm{~mm}$; (3) T1WIcor: minimum TR, minimum TE, FOVAP 125 mm, RL 183 mm, FH $230 \mathrm{~mm}$, layer thickness $6 \mathrm{~mm}$, layer number 18, and layer spacing $1 \mathrm{~mm}$; (4) T2WI- Fairtrade: TR $6000 \mathrm{~ms}$, TE $120 \mathrm{~ms}$, FOVAP $230 \mathrm{~mm}$, RL $208 \mathrm{~mm}$, FH $125 \mathrm{~mm}$, layer thickness $6 \mathrm{~mm}$, layer number 18 , and layer spacing $1 \mathrm{~mm}$.

2.5. Image Fusion Steps. Fusion can be performed after the image is matched. IR represents infrared image, while VIS represents visible-light image. First, the low-frequency part fusion is actually two steps: block division and block selection. The low-frequency components in the source image can reflect its characteristics relatively, and the low-frequency components of the two images actually have similar parts. Then we use structural similarity as an index to evaluate fusion. To obtain and maintain the relatively complete structure of the result, it is necessary to combine the structural similarity and low-frequency components as its fitness function during particle swarm optimization. Take the structural similarity of $L_{I} R, L_{F}$ as an example:

$$
\operatorname{SSIM}\left(L_{\mathrm{IR}}, L_{F}\right)=\frac{\left(2 u_{L_{\mathrm{IR}}} u_{L_{F}}+C_{1}\right)\left(2 \sigma_{L_{\mathrm{IR}}}, L_{F}+C_{2}\right)}{\left(u_{L_{\mathrm{IR}}}^{2}+u_{L_{F}}^{2}+C_{1}\right)\left(\sigma_{L_{\mathrm{IR}}}^{2}+\sigma_{L_{F}}^{2}+C_{2}\right)}
$$

In formula (1), $u_{L_{I R}}$ is the average value of the lowfrequency components in the infrared image, $u_{L_{F}}$ is the average value of the low-frequency part of the image, and $\sigma_{L_{F}}$ and $\sigma_{L_{\mathrm{IR}}}$ are the regional variances of the two. $\sigma_{L_{\mathrm{IR}}}, L_{F}$ are the joint variances, where the size of this part is $11 \times 11$ Gaussian window. To simplify the calculation, both $\mathrm{C} 1$ and $\mathrm{C} 2$ are set to 0 . The structural similarity of $L_{\mathrm{IR}}$ and $L_{\mathrm{VIS}}$ and $L_{F}$ is

$$
\operatorname{SSIM}\left(L_{\mathrm{IR}}, L_{\mathrm{VIS}}, L_{F}\right)=\frac{1}{2}\left[\operatorname{SSIM}\left(L_{\mathrm{IR}}, L_{F}\right)+\operatorname{SSIM}\left(L_{\mathrm{VIS}}, L_{F}\right)\right] .
$$

The particle swarm algorithm is a random search optimization algorithm that continuously updates the particle position through two aspects: the particle optimal solution and the swarm optimal solution. Use this to find the optimal value. The particle speed update formula of the particle swarm algorithm is as follows:

$$
v_{i j}(t+1)=\omega v_{i j}(t)+c_{1} r_{1 j}(t)\left[p_{i j}(t)-x_{i j}(t)\right]+c_{2} r_{2 j}(t)\left[p_{g j}(t)-x_{i j}(t)\right]
$$

The particle position is determined by the following formula:

$$
x_{i j}(t+1)=x_{i j}(t)+v_{i j}(t+1) .
$$

For an $N \times N$ region, the gradient energy method is

$$
G=\sum_{i=1}^{N} \sum_{j=1}^{N}\left[f_{x}(i, j)^{2}+f_{y}(i, j)^{2}\right]
$$

where $f_{x}(i, j)$ represents the gradient change in the horizontal direction and $f_{x}(i, j)$ represents the gradient change in the vertical direction. After the boundary block is processed as described above, there will be a result $\overline{L_{F}}$ of $L_{F}$. In order to solve this problem, the neighbourhood coefficient difference is selected and the label map is used to merge the high-frequency components. The neighbourhood coefficient difference can reflect the deviation degree between the part where the pixel is located and the average of this part in the 
image. The larger the coefficient, the higher the deviation degree. Set the image centre point to $(i, j)$, where the window size is $N \times N$ area:

$$
\left\|e_{X}(i, j)\right\|_{N \times N}=\sum_{m=-N-1 / 2}^{m=N-1 / 2} \sum_{n=-N-1 / 2}^{n=N-1 / 2}\left\|e_{X}(i+m, j+n)-\overline{e_{x}(i, j)_{N \times N}}\right\| .
$$

Formula (6) is the neighbourhood coefficient difference formula and $\overline{e_{x}(i, j)_{N \times N}}$ represents the absolute average value of high-frequency components in $N \times N$.

2.6. MRS Data Processing Principle. Using MRS metabolites to calculate temperature, the relationship between temperature $(T)$ and water proton chemical shift $\left(\mathrm{CSH}_{2} \mathrm{O}\right)$ is shown in

$$
T=T_{\text {ref }}+k\left(\mathrm{CSH}_{2} \mathrm{O}-\mathrm{CS}_{\mathrm{ref}}\right) .
$$

Among them, the reference chemical shift $\mathrm{CS}_{\mathrm{ref}}$ is the chemical shift of the temperature-independent compound, $k$ is the proportional constant, and $T_{\text {ref }}$ is the reference temperature. Under standard conditions, the chemical shift of hydrogen protons is $4.7 \mathrm{ppm}$. It is known that the chemical shift of water protons will change under the influence of temperature. Because the chemical shift of the reference substance is constant, the effect of temperature on water protons can be converted into an effect on $\mathrm{CSH}_{2} \mathrm{O}-\mathrm{CS}_{\text {ref }}$.

$$
T=37+100\left(\mathrm{CSNAA}_{\left.-\mathrm{CSNAA}_{\mathrm{ref}}\right)},\right.
$$

where CSNAA $_{\text {ref }}$ is the reference CSNAA, which can be obtained through experiments. It is reported in the literature that this formula is applied to the study of the infarct area and ischemic penumbra of patients with acute cerebral ischemia. The temperature formula is $T=37+100$ (CSNAA-2.035) after detecting the brain temperature of 20 normal people. In our study, this formula was applied to the calculation of brain temperature of drug addicts. Calculate the subjects' absolute brain temperature and compare the differences in brain temperature before and after visual stimulation.

2.7. Scale Evaluation. The Hamilton Anxiety Rating Scale and Depression Rating Scale were used to measure the emotional state of all subjects to exclude patients with severe anxiety and depression. Anxiety evaluation criteria are total score $\geq 29$ points, denoting that there may be severe anxiety; total score $\geq 21$ points, denoting that there must be obvious anxiety; total score $\geq 14$ points, denoting that there must be anxiety; total score $\geq 7$ points, denoting that there may be anxiety; total score $<7$ points, denoting that there are no anxiety symptoms. Depression evaluation standards are total score $<7$ points, denoting normal; total score of $7-17$ points, denoting that there may be depression; total score of 17-24 points, denoting that there must be depression; total score $>24$ points, denoting severe depression.

\section{Results}

3.1. Participant Characteristics. In the heroin experiment, the average age of heroin addicts was $35.93 \pm 5.65$ years, and the length of education was $7.43 \pm 1.74$ years; the average age of the normal group was $34.31 \pm 9.45$ years, and the length of education was $7.93 \pm 1.39$ years. There was no statistical difference in average age and years of education $(P>0.05)$ (see Table 1).

In the methamphetamine experiment, the average age of methamphetamine-dependent persons was $31.17 \pm 5.01$ years, and the length of education was $8.33 \pm 2.31$ years; the average age of the normal group was $31.11 \pm 5.86$ years, and the length of education was $9.11 \pm 1.69$ years. There was no statistical difference in average age and years of education $(P>0.05)$ (see Table 2).

\subsection{Brain Temperature Results}

3.2.1. Results of Heroin Experiment Brain Temperature. Table 3 compares the average brain temperature of the prefrontal cortex in the heroin group and the normal group before and after visual stimulation [3]. The paired $t$-test was used for comparison between the two groups. The average brain temperature of the prefrontal cortex after visual stimulation in the heroin group and the normal group was higher than that before visual stimulation, but there was no statistical difference between the two $(P>0.05)$. Table 4 compares the average brain temperature of the prefrontal cortex between the heroin group and the normal group before and after visual stimulation.

\subsubsection{Brain Temperature Results of Methamphetamine} Experiment. Table 5 compares the average brain temperature of the prefrontal cortex, the left hippocampus, and the right hippocampus before and after visual stimulation in the methamphetamine group and the normal group.

Table 6 shows the comparison of the average brain temperature of the prefrontal cortex, the left hippocampus, and the right hippocampus between the methamphetamine group and the normal group before and after visual stimulation. The independent sample $t$-test was used for comparison between the two groups. Compared with the normal group, the average brain temperature of the left hippocampus of the methamphetamine group was reduced, and the average brain temperature of the left hippocampus of the two groups had statistically significant changes $(P<0.05)$.

The prefrontal cortex spectrum curves of the heroin group before and after visual stimulation and the matched normal group are shown in Figures 4 and 5. The spectrum curves of the prefrontal cortex, left hippocampus, and right hippocampus of the methamphetamine group and the matched normal group are shown in Figures 6 and 8. The figure shows NAA chemical shift values before and after visual stimulation in the heroin group, methamphetamine group, and normal group. 

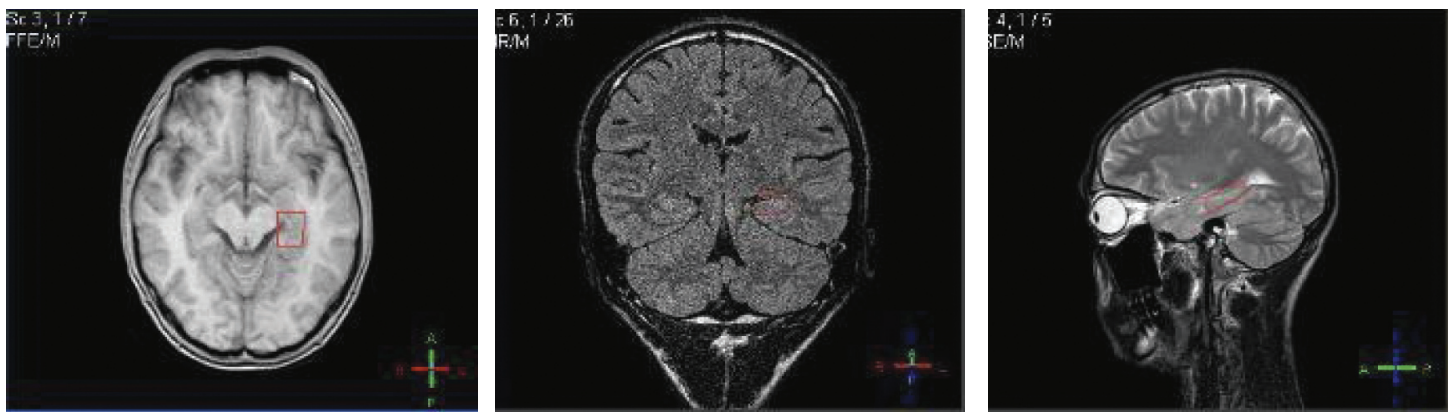

FIGURE 2: Left hippocampus location map.
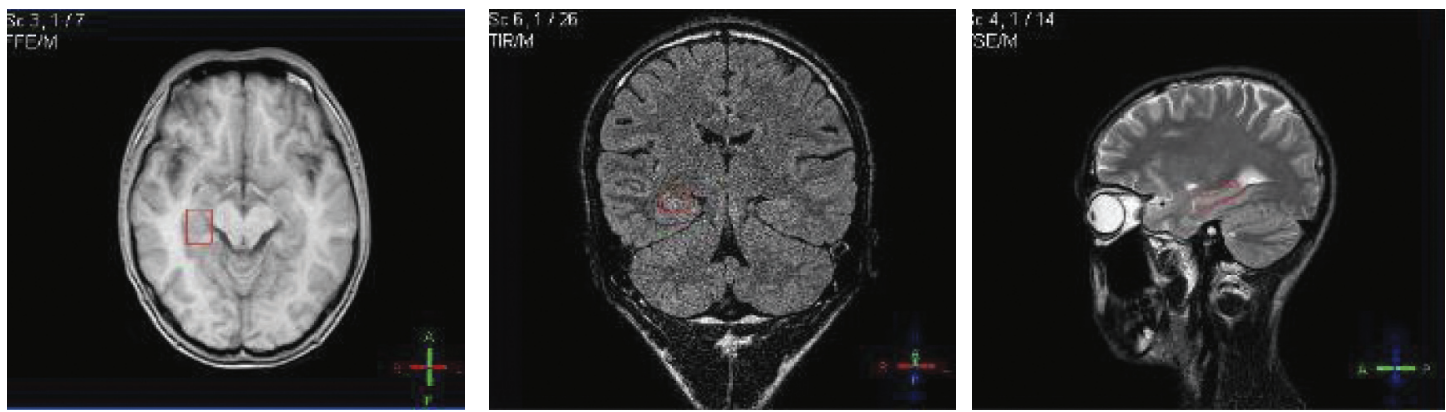

FIgURE 3: Right hippocampus location map.
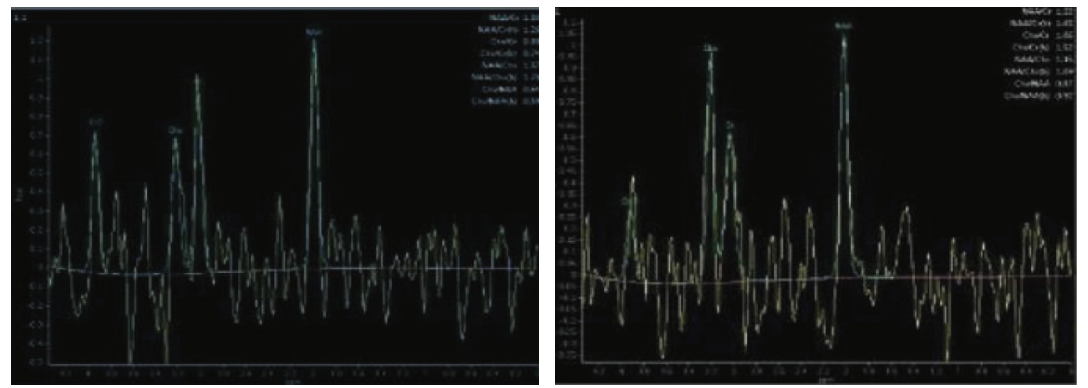

FIgURE 4: Prefrontal lobe spectrum curve before and after stimulation in the HD group.
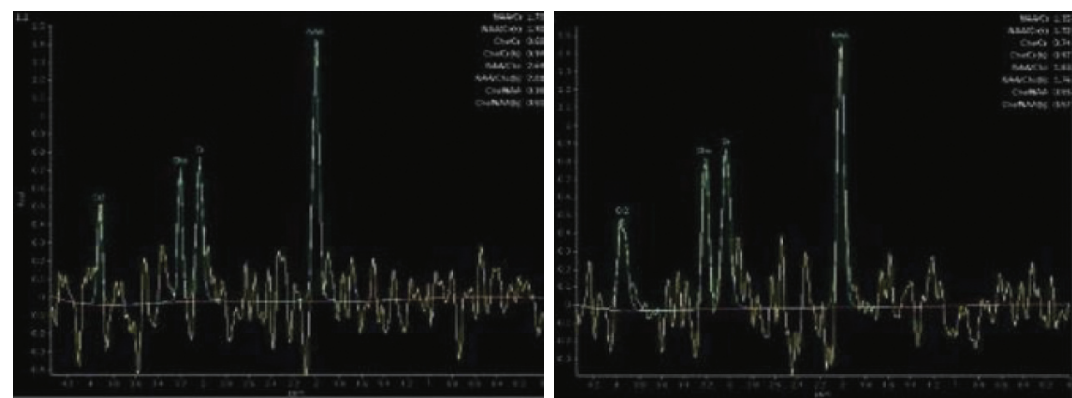

Figure 5: Prefrontal lobe spectrum curve before and after stimulation in the $\mathrm{HC1}$ group.

\section{Discussion}

Drug dependence is a chronic brain disease with the characteristics of recurrent attacks. Drug abuse not only endangers the physical and mental health and family happiness of dependents but also causes a series of social problems and brings serious harm to society. The mechanism of drug dependence and addiction has not been studied clearly, which brings difficulties to clinical withdrawal treatment. MRS can noninvasively study the material 



Figure 6: Prefrontal lobe spectrum curve before and after stimulation in the MD group.
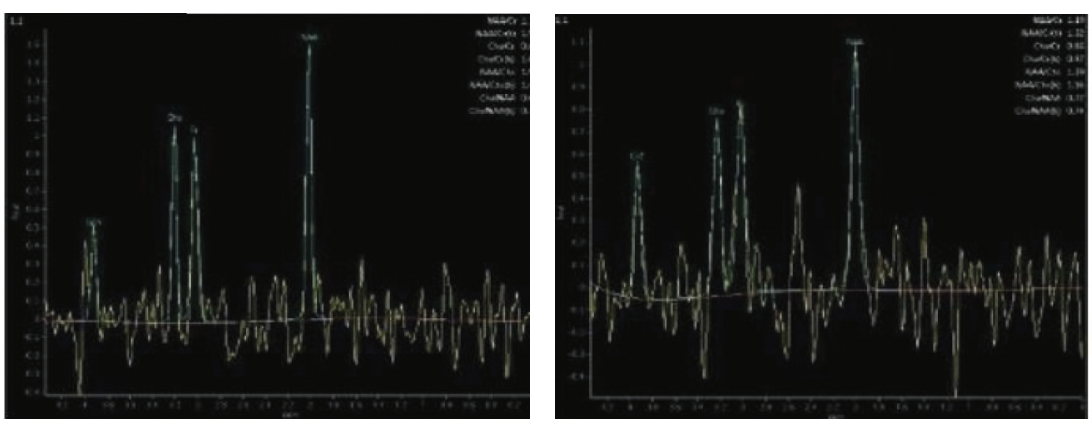

Figure 7: Left hippocampal spectrum curve before and after stimulation in the MD group.
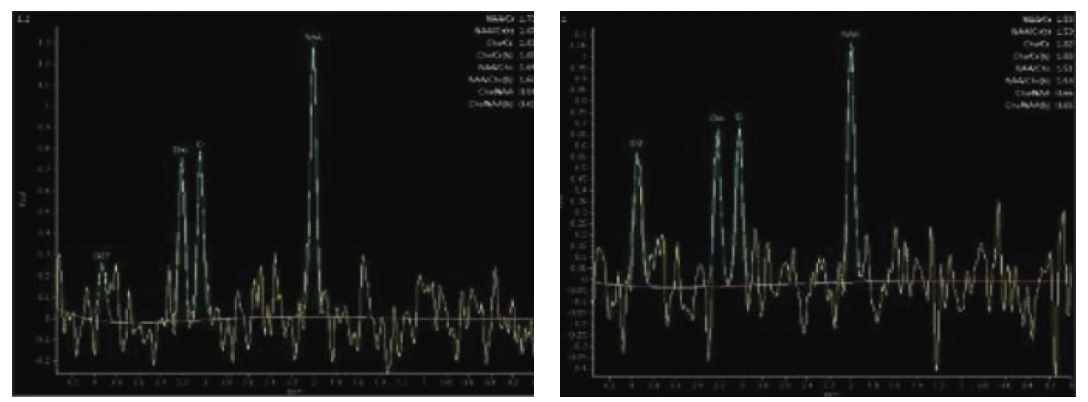

Figure 8: Prefrontal lobe spectrum curves before and after stimulation in HC2 group.

metabolism of human internal tissues and organs and can be used as a quantitative method to detect the concentration of various metabolites in the brain. In recent years, MRS measurement of brain temperature has been reported in the research of stroke and traumatic brain injury, and there has not been any research on the application of MRS measurement of brain temperature in the field of drug addiction. This study uses 1HMRS to measure brain temperature to study the changes in brain temperature of heroin and methamphetamine addicts in the prefrontal cortex and bilateral hippocampus during visual stimulation of drug-related cues, aiming to use brain temperature as a quantitative indicator to reveal drug addiction and try to use it as an indicator to quantitatively judge the psychological cravings of drug-dependent persons and the degree of drug dependence [4].
4.1. Principle of MRS Measuring Brain Temperature. There are many ways to measure temperature in the biomedical field. Magnetic resonance temperature imaging can measure the temperature distribution of deep tissues. It has good tissue resolution and noninvasiveness. It is one of the most ideal methods for temperature measurement and monitoring. The magnetic resonance parameters commonly used in temperature imaging include longitudinal relaxation time constant (T1), proton resonance frequency shift (PRFS), apparent diffusion coefficient of water molecules (ADC), and intermolecular quantum coherence (iMQC). Among these methods, the magnetic resonance temperature imaging based on the principle of PRFS is the most widely used. This is because the method has a better temporal and spatial resolution, the proton resonance frequency has an ideal linear relationship with temperature changes, the linear 
TABle 1: Demographic data of heroin experiment subjects.

\begin{tabular}{lccc}
\hline Grouping & Heroin group & Normal control group & $t$ \\
\hline Age & $35.93 \pm 5.65$ & $34.31 \pm 9.45$ & 0.576 \\
Education years (years) & $7.43 \pm 1.74$ & $7.93 \pm 1.39$ & -0.890 \\
Average daily dosage of heroin (g) & $0.26 \pm 0.16$ & - & - \\
Heroin use time (years) & $8.57 \pm 4.50$ & - & - \\
Withdrawal time (month) & $1.57 \pm 0.51$ & - & - \\
Anxiety state & $6.86 \pm 3.13$ & - & - \\
Depression & $8.36 \pm 3.82$ & - & - \\
\hline
\end{tabular}

TABLE 2: Demographic data of participants in the methamphetamine experiment.

\begin{tabular}{|c|c|c|c|c|}
\hline Grouping & Methamphetamine group & Normal control group & $t$ & $P$ \\
\hline Age & $31.17 \pm 5.01$ & $31.11 \pm 5.86$ & 0.023 & 0.982 \\
\hline Education years & $8.33 \pm 2.31$ & $9.11 \pm 1.69$ & -0.851 & 0.405 \\
\hline Average daily consumption of methamphetamine (g) & $0.19 \pm 0.11$ & - & - & - \\
\hline Methamphetamine use time & $3.08 \pm 2.84$ & - & - & - \\
\hline Withdrawal time & $1.92 \pm 0.90$ & - & - & - \\
\hline Anxiety state & $5.92 \pm 3.42$ & - & - & - \\
\hline Depression & $5.08 \pm 1.83$ & - & - & - \\
\hline
\end{tabular}

TABLE 3: Comparison of the average brain temperature of the prefrontal cortex before and after visual stimulation in the two groups.

\begin{tabular}{lcrrr}
\hline Grouping & Before visual stimulation $\left({ }^{\circ} \mathrm{C}\right)$ & After visual stimulation $\left({ }^{\circ} \mathrm{C}\right)$ & $t$ & $P$ \\
\hline Heroin group & $34.48 \pm 0.92$ & $34.61 \pm 0.92$ & 0.379 \\
Normal control group & $34.01 \pm 0.93$ & $34.70 \pm 1.06$ & 0.711 \\
\hline
\end{tabular}

TABLE 4: Comparison of the average brain temperature of the prefrontal cortex before and after visual stimulation between the two groups.

\begin{tabular}{lcc}
\hline Grouping & Before visual stimulation $\left({ }^{\circ} \mathrm{C}\right)$ & After visual stimulation $\left({ }^{\circ} \mathrm{C}\right)$ \\
\hline Heroin group & $34.48 \pm 0.92$ & $34.61 \pm 0.92$ \\
Normal control group & $34.01 \pm 0.93$ & $34.70 \pm 1.06$ \\
$t$ value & 1.378 & -0.235 \\
$P$ value & 0.179 & 0.861 \\
\hline
\end{tabular}

TABLE 5: Comparison of the average brain temperature of the prefrontal cortex and bilateral hippocampus before and after visual stimulation in the two groups.

\begin{tabular}{lccccc}
\hline \multirow{3}{*}{ Methamphetamine group } & Prefrontal cortex & $34.67 \pm 0.72$ & $34.87 \pm 0.91$ & -0.642 & 0.534 \\
& Left hippocampus & $34.56 \pm 0.61$ & $34.44 \pm 0.54$ & 0.736 & 0.477 \\
& Right hippocampus & $34.49 \pm 0.46$ & $34.41 \pm 0.19$ & 0.523 & 0.612 \\
\hline \multirow{3}{*}{ Normal group } & Prefrontal cortex & $34.92 \pm 1.24$ & $34.78 \pm 0.96$ & 0.464 & 0.655 \\
& Left hippocampus & $34.74 \pm 0.14$ & $34.92 \pm 0.38$ & -1.336 & 0.218 \\
& Right hippocampus & $34.53 \pm 0.45$ & $34.56 \pm 0.23$ & -0.148 & 0.886 \\
\hline
\end{tabular}

relationship does not vary with tissue, the environment changes and changes, and it is often used to measure the absolute temperature of the tissue. NAA is a chemical substance rich in brain content, and its content in the brain can be detected using 1HMRS. Due to the larger peak of NAA in the adult brain, NAA can provide a more accurate reference compared with other endogenous substances (such as choline complex and total creatine). NAA metabolism in the brain will change after drug addiction. Therefore, in our experiment, we selected the NAA peak as the research object to explore the changes in brain temperature of heroin and methamphetamine-dependent patients.
4.2. Self-Balancing Mechanism of Body Temperature and Brain Temperature. The body temperature control centre is located in the preoptic nucleus at the back of the hypothalamus, which can balance heat production and heat dissipation by activating different pathways. Shudder, arteriovenous shunt, vasoconstriction, thermogenesis of fat, and adaptive behaviour are the main mechanisms to maintain a constant core temperature $\left(36-37^{\circ} \mathrm{C}\right)$ to meet environmental changes and the needs of the body. However, body temperature is not evenly distributed in all parts of the body. To ensure that the body temperature remains constant, peripheral organs and skin play a vital role in the redistribution of heat generated by the body to maintain the body 
TABLE 6: Comparison of the average brain temperature of the prefrontal cortex and bilateral hippocampus before and after visual stimulation between the two groups.

\begin{tabular}{|c|c|c|c|c|c|c|}
\hline \multirow{2}{*}{ Grouping } & \multicolumn{2}{|c|}{ Prefrontal cortex $\left({ }^{\circ} \mathrm{C}\right)$} & \multicolumn{2}{|c|}{ Left hippocampus $\left({ }^{\circ} \mathrm{C}\right)$} & \multicolumn{2}{|c|}{ Right hippocampus $\left({ }^{\circ} \mathrm{C}\right)$} \\
\hline & Before & After & Before & After & Before & After \\
\hline Methamphetamine group & $34.67 \pm 0.72$ & $34.87 \pm 0.9$ & $34.56 \pm 0.6$ & $34.44 \pm 0.5$ & $34.49 \pm 0.4$ & $34.41 \pm 0.1$ \\
\hline Normal group & $34.92 \pm 1.24$ & $34.78 \pm 0.9$ & $34.74 \pm 0.1$ & $34.92 \pm 0.3$ & $34.53 \pm 0.4$ & $34.56 \pm 0.2$ \\
\hline$t$ & -0.594 & 0.217 & -1.021 & -2.236 & -0.029 & -1.616 \\
\hline$P$ & 0.56 & 0.831 & 0.327 & $0.036^{*}$ & 0.837 & 0.123 \\
\hline
\end{tabular}

temperature below the core temperature. When the environment temperature changes drastically, the temperature gradient between the core and the peripheral tissue can be changed from -4 to $+2^{\circ} \mathrm{C}$ to maintain the core temperature relatively stable. The above-mentioned thermoregulation process is called thermoregulation response [5].

\subsection{Effect of Psychoactive Drugs and Drug-Related Visual} Stimulation on Brain Temperature. Most psychoactive drugs change brain temperature by affecting brain metabolism and cerebral blood flow. Drug-induced brain temperature changes can affect the activity and function of neurons. We usually regard brain temperature as a fixed constant temperature parameter, which depends on the balance of brain metabolism and heat dissipation. Studies have shown that changes in the body's activity state, environmental stimuli, and neuromotivated behaviour will cause relatively large physiological fluctuations in brain temperature. Most psychoactive drugs can affect brain metabolism and the state of peripheral blood vessels and cerebral blood vessels, so the brain temperature can fluctuate around the normal baseline level $\left(37^{\circ} \mathrm{C}\right)$. Contrary to transient physiological fluctuations, the brain temperature changes induced by psychoactive drugs depend on the dosage and usage of the drugs, and the drugs themselves can interfere with the body's normal physiological processes. It is generally believed that the brain temperature of healthy warm-blooded animals is relatively stable, close to $37^{\circ} \mathrm{C}$. Data from a large number of animal experiments show that exposure to different environmental conditions or different types of neuromotivated behaviours can cause dramatic fluctuations in brain temperature. There are reports in the literature showing that, by implanting miniature thermocouple electrode sensors into different brain regions of rats to study the changes in brain temperature of freely active male rats in a waking state, it is found that the brain temperature of rats in sleep state can drop to $35^{\circ} \mathrm{C}$. The peak brain temperature of male rats in mating state can reach $39.5^{\circ} \mathrm{C}$ at the moment of ejaculation. The recording of rat brain temperature is relatively simple, while reports on direct measurement of human brain temperature are rare or only limited to neurological patients [6].

State dependence is particularly important for drug abuse. It usually refers to the fact that the drug user is in a state of psychophysiological activation and in a relatively closed environment, thereby reducing brain heat dissipation. Under such conditions, amphetamine-like stimulants can induce pathological brain hyperthermia (brain temperature $>40^{\circ} \mathrm{C}$ ), which is related to the leakage of the blood-brain barrier and abnormal brain cell structure. The subjects selected for this study are heroin and methamphetamine-dependent persons. Although they are currently in a short-term withdrawal state, their medical history of drug use before withdrawal is long. Among them, the average history of heroin use in the heroin group was $8.57 \pm 4.50$ years, and the average history of methamphetamine in the methamphetamine group was $3.08 \pm 2.84$ years, so it is speculated that the blood-brain barrier and brain cells may be abnormal. In our experiments, the brain temperatures of the prefrontal lobes and bilateral hippocampus of heroin and methamphetamine-dependent patients before and after visual stimulation were lower than $37^{\circ} \mathrm{C}$, which may be related to the laboratory environment temperature. Studies have shown that general anaesthetics can cause the body's body temperature to drop rapidly and severely and cause serious damage to the body's body temperature regulation mechanism. For this reason, the body is particularly sensitive to the influence of external temperature. Because the magnetic resonance (MR) equipment requires the ambient temperature to be maintained at about 22 degrees Celsius, we monitored that the ambient temperature basically fluctuates between $19^{\circ} \mathrm{C}$ and $23^{\circ} \mathrm{C}$ in the experiment, which may increase brain heat dissipation and make the subject's brain temperature below $37^{\circ} \mathrm{C}$.

\subsection{Reasons for Changes in the Temperature of the Prefrontal} Cortex and Hippocampus. Brain temperature depends on the interaction of brain oxygen metabolism rate and cerebral blood flow. Visual stimulation can increase cerebral blood flow and cerebral oxygen metabolism rate at the same time. The fMRI studies in the field of predrug addiction show that drug-dependent patients will be activated under the induction of environmental and drug-related cues. These brain regions include the nucleus accumbens, prefrontal lobe, amygdala, hippocampus, precuneus, orbit the frontal gyrus, lower corpus callosum, and cerebellum, and other brain regions are mainly responsible for rewards, cravings, emotional processing, working memory, motivation processing, decisionmaking, executive control, and sensory integration. We used drug-related visual stimuli to study the prefrontal cortex temperature of heroin and methamphetamine-dependent patients in the short-term withdrawal group. The results showed that visual stimulation did not cause significant changes in the prefrontal cortex brain temperature, but in the previous experiments, the prefrontal cortex belongs to the positive BOLD brain activation area [7]. 
The NAA/Cr value of the hippocampus of heroin addicts decreased, suggesting that long-term heroin smoking can cause neuron damage in the hippocampus of addicts. In recent years, the abuse of new drugs has become more common, and the number of users of methamphetamine in China has been increasing year by year. Studies have shown that ice has a more serious impact on the central system than heroin. We studied the changes in the hippocampal temperature of both sides of the hippocampus before and after visual stimulation in the methamphetamine addicts in the short-term withdrawal group and found that, compared with the normal group, the temperature of the left hippocampus decreased after visual stimulation, and the temperature of the left hippocampus changed in the two groups. There is a statistical difference $(P<0.05)$. Studies have shown that after long-term drug users undergo compulsory detoxification when they are exposed to drug-related environmental cues (such as visual stimulation), and their corresponding addictive brain regions will still be activated. Therefore, we speculate that the memory of methamphetamine users is awakened and excitability is increased under visual stimulation, and the blood flow of the left hippocampus increases but the oxygen metabolism rate does not increase significantly, which causes the brain temperature of the left hippocampus to decrease. MRS studies have shown that the temperature of the occipital lobe of the brain drops after a longer period of visual stimulation (stimulation usually lasts several minutes). In our experiment, the time to scan the hippocampal MRS is 7 minutes, and the visual stimulation time is longer, so it can also explain the reason for the temperature drop in the left hippocampus.

\section{Conclusion}

(1) When heroin and methamphetamine-dependent persons in a short-term withdrawal state were given drug-related visual cues stimulation, the brain temperature of their prefrontal cortex did not change significantly, indicating that visual stimulation has a weak effect on the change of prefrontal cortex brain temperature.

(2) The brain temperature of the left hippocampus of the methamphetamine addicts was lower than the normal group when visual stimulation was given, indicating that the methamphetamine-related visual cues stimulated the drug memory of the methamphetamine addicts, resulting in a sense of excitement. It is speculated that the blood flow in the left hippocampus increased, and the increase in oxygen metabolism rate is not obvious, leading to a decrease in the brain temperature of the left hippocampus, while visual stimulation has a weaker effect on the change of the right hippocampus.

\section{Data Availability}

The data used to support the findings of this study are available from the corresponding author upon request.

\section{Conflicts of Interest}

The authors declare that there are no conflicts of interest regarding the publication of this article.

\section{References}

[1] M. M. Baig, H. Gholamhosseini, and M. J. Connolly, "Mobile healthcare applications: system design review, critical issues and challenges," Australasian Physical \& Engineering Sciences in Medicine, vol. 38, no. 1, pp. 23-38, 2015.

[2] M. R. Vafaeinasab, M. H. Farahzadi, O. M. Razaghi, R. A. Fallahzadeh, M. H. Lotfi, and S. Akhondzadeh, "Investigation of affecting factors on persistence in the treatment of patients under methadone maintenance therapy in addiction therapy centers, yazd-Iran," Health, vol. 7, no. 5, pp. 606-616, 2015.

[3] B. Loi, J. M. Corkery, H. Claridge et al., "Deaths of individuals aged 16-24 years in the UK after using mephedrone," Human Psychopharmacology: Clinical and Experimental, vol. 30, no. 4, pp. 225-232, 2015.

[4] R. Kim, K.-J. Lee, and Y.-J. Choi, "Mobile phone overuse among elementary school students in Korea," Journal of Addictions Nursing, vol. 26, no. 2, pp. 81-85, 2015.

[5] U. Kirchmayer, M. Davoli, A. D. Verster, L. Amato, and C. A. Perucci, "A systematic review on the efficacy of naltrexone maintenance treatment in opioid dependence," $A d$ diction, vol. 92, no. 10, pp. 1241-1249, 2015.

[6] O. Lortholary, C. Renaudat, C. Renaudat et al., "The risk and clinical outcome of candidemia depending on underlying malignancy," Intensive Care Medicine, vol. 43, no. 5, pp. 652-662, 2017.

[7] Z. R. Steelman and A. A. Soror, "Why do you keep doing that? the biasing effects of mental states on it continued usage intentions," Computers in Human Behavior, vol. 73, no. 8, pp. 209-223, 2017. 